\title{
Constructions of expression vectors of polyhydroxybutyrate-co-hydroxyvalerate (PHBV) and transient expression of transgenes in immature oil palm embryos.
}

\begin{abstract}
Polyhydroxybutyrate-co-hydroxyvalerate (PHBV) is a polyhydroxyalkanoate (PHA) bioplastic group with thermoplastic properties is thus high in quality and can be degradable. PHBV can be produced by bacteria, but the process is not economically competitive with polymers produced from petrochemicals. To overcome this problem, research on transgenic plants has been carried out as one of the solutions to produce PHBV in economically sound alternative manner. Four different genes encoded with the enzymes necessary to catalyze PHBV are bktB, phaB, phaC and tdcB. All the genes came with modified CaMV 35S promoters (except for the tdcB gene, which was promoted by the native CaMV $35 \mathrm{~S}$ promoter), nos terminator sequences and plastid sequences in order to target the genes into the plastids. Subcloning resulted in the generation of two different orientations of the tdcB, pLMIN (left) and pRMIN (right), both 17.557 and $19.967 \mathrm{~kb}$ in sizes. Both plasmids were transformed in immature embryos (IE) of oil palm via Agrobacterium tumefaciens. Assays of GUS were performed on one-week-old calli and $90 \%$ of the calli turned completely blue. This preliminary test showed positive results of integration. Six-months-old calli were harvested and RNA of the calli were isolated. RT-PCR was used to confirm the transient expression of PHBV transgenes in the calli. The bands were 258, 260, 315 and $200 \mathrm{bp}$ in size for bktB, phaB, phaC and tdcB transgenes respectively. The data obtained showed that the bktB, phaB, phaC and tdcB genes were successfully integrated and expressed in the oil palm genome.
\end{abstract}

Keyword: PHBV; Plamids; Transgene; Agrobacterium; Oilpalm. 